\title{
Large angle flexure pivot development for future science payloads for space applications
}

\author{
Peter Spanoudakis ${ }^{l}$, Lionel Kiener $^{l}$, Florent Cosandier $^{l}$, Philippe Schwab ${ }^{l}$, \\ Laurent Giriens ${ }^{l}$, Johan Kruis ${ }^{1}$, Daniel Grivon ${ }^{1}$, Georgia Psoni $^{2}$, Christos Vrettos ${ }^{2}$ \\ and Nabil Bencheikh ${ }^{3}$ \\ ${ }^{1}$ CSEM SA, Jaquet-Droz 1, CH-2002 Neuchâtel, Switzerland \\ ${ }^{2}$ Heron Engineering, 2 Asklipiou St. \& Mesolongiou, Nea Ionia, 14231, Athens, Greece \\ ${ }^{3}$ Cedrat Technologies, 59 Chemin du Vieux Chêne 38246 Meylan Cedex France
}

\begin{abstract}
An innovative design of a Large Angle Flexure Pivot (LAFP) is described. It combines the advantages of flexure mechanisms while surpassing one of their few flaws, small displacement strokes. The LAFP design exceeds these angular limitations to reach a deflection of $180^{\circ}$ $\left( \pm 90^{\circ}\right)$. The centre shifts laterally by less than $\pm 35 \mu \mathrm{m}$ throughout the full rotation range. The LAFP is meant to be mounted in pairs, coaxially and with the payload between them. The intended application of the LAFP is to angularly guide an optical component in a space environment for future science missions operating in a cryogenic environment. A dedicated performance test bench was developed and manufactured to test the pivot characteristics notably the lateral shift using Eddy current sensors. The test bench incorporates a representative dummy payload for mass and inertia. Extensive FEM analysis has been performed to validate the design at component level and further analysis with the pivots mounted with a representative payload on a test bench for random vibration, shock and thermal cycling environment. The second test bench for the vibration and shock tests has been manufactured incorporating a simplified launch locking device. The performance tests have confirmed a lateral shift of less than $\pm 35 \mu \mathrm{m}$ over an angular range of $\pm 90^{\circ}$. The pivots have been successfully tested and survived vibration loads for high level sine at $24 \mathrm{~g}$ and random vibration at 12 grms in all three directions.
\end{abstract}

\section{Introduction}

An innovative design of a Large Angle Flexure Pivot (LAFP) has been developed (Fig. 1) combining the advantages of flexure mechanisms - no friction, no backlash, no need for lubricant, no wear - while surpassing one of their few flaws, small displacement strokes. These are usually comprised between $10^{\circ}$ and $20^{\circ}$ for typical flexible angular pivots. The LAFP design exceeds these angular limitations to a range where previously only ball bearings would be adequate. The angular stroke can reach a deflection of $180^{\circ}\left( \pm 90^{\circ}\right)$ and can easily meet a lifetime of three million cycles. If the stroke is limited to $\pm 70^{\circ}$, infinite operational lifetime is obtained. 


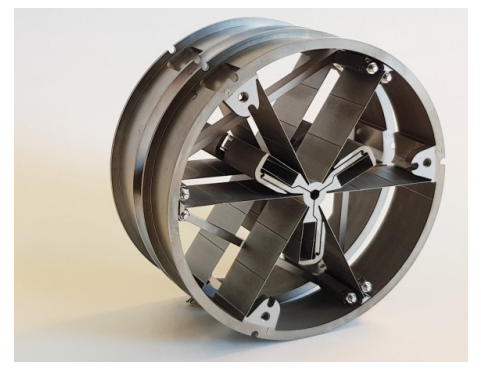

Fig. 1. LAFP Engineering Model

The LAFP is $120 \mathrm{~mm}$ in diameter, $60 \mathrm{~mm}$ in length and weighs less than $500 \mathrm{~g}$. A pair of pivots has been sized to carry a payload of $1.8 \mathrm{~kg}$ (including interfaces) and offers a low rotational stiffness while ensuring high lateral and transverse stiffness. In order to meet future science missions in a cryogenic environment, they can operate in a temperature ranging from $-140^{\circ} \mathrm{C}$ to $+65^{\circ} \mathrm{C}$. The centre shifts laterally by less than $10 \mu \mathrm{m}$ throughout the full rotation range. The LAFP is mounted in pairs, coaxially with the payload between them. The intended application of the LAFP is to angularly guide an optical component in a space environment.

\section{Large angle flexure pivot concept}

Within the diversity of flexure mechanisms, many are dedicated to very high precision, but only few go beyond the inherently short limit of their displacements strokes. CSEM surpassed this restriction by developing a flexure pivot with a very large stroke, which is endowed with excellent performance [1 and 2]. With a long heritage in the design of compliant mechanisms, CSEM has especially conceived the iconic Butterfly pivot [3], which offers $\mathrm{a} \pm 15^{\circ}$ stroke. Examples of large stroke flexure pivot exist in literature. One should notice the FLEX 16 [4], whose stroke is $\pm 90^{\circ}$. However, its centre shift is high and stiffness is low. Another example is the infinity-hinge [5] which proposes large strokes of $\pm 45^{\circ}$ while preserving adequate radial stiffness.

In the presented design, there are two identical half-pivots assembled in a series configuration (Fig. 2a) and linked by a coupling triangle. Each of the half-pivots has six main radial leaf springs and six coupling leaf springs. The main leaf springs are laid out by pairs serially linking the rim to the coupling triangle, via three rigid portions. These are linked by pairs through coupling stages, which are composed of two short parallel leaf springs. When the pivot is rotated (Fig. $2 b$ ), the rigid portions move away from the central axis so as to let the main leaf springs shorten. It is not possible to rigidly link the portions to each other, as the stroke would be diminished. Conversely, not linking these portions would result in blade warping and a drop in stiffness. The coupling stages link the portions correctly, permitting the desired synchronous and radial movement.
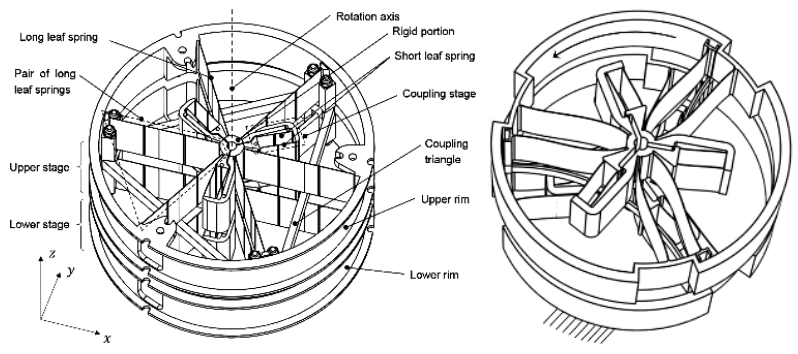

Fig. 2. (a) LAFP at rest position and (b) in deflected position 


\subsection{Radial leaf springs deformation and shortening}

Fig. 3a illustrates the shortening of a long leaf spring, whose left side is fixed to the rim and right side rotates with the rigid portion around the $\mathrm{z}$-axis by an angle $\theta$. It results in a shortening $\lambda$ from which one can calculate $\mathrm{s}$ the stroke of the coupling stage. Regardless of the rotation direction of the LAFP, its coupling stages always travel in the same direction. Thus, it is possible to preload the coupling stage to obtain a stroke of $-\mathrm{s} / 2$ to $+\mathrm{s} / 2$, instead of 0 to $+\mathrm{s}$. This reduces the maximum stress in the stages and enhances their performance. The preload gap (see Fig. 3b) is adjusted as part of the assembly process.
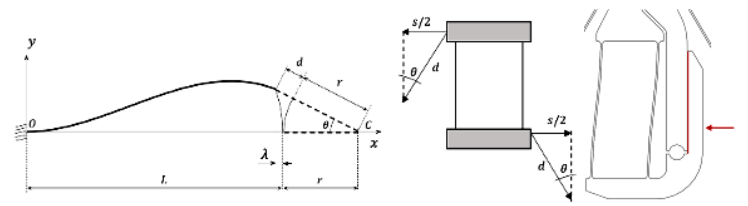

Fig. 3. (a) Shortening of the long leaf springs, (b) preload of coupling stage

\subsection{FEA performance evaluation}

Extensive FEM analysis has been performed to validate the design at component level and further analysis with the pivots mounted with a representative payload on a test bench for random vibration, shock and thermal cycling environment. The preliminary FEA was performed by CSEM with COMSOL and in a second phase by Heron using NASTRAN. Generally, simulating the LAFP is complex due to very large deformations exerted on leaf springs with high slenderness ratios and the preload. In COMSOL, the simulations were realized on a model with rigid connectors, representing the structural parts. The simulations concerned static (torque, stiffness, stress, centre shift, buckling) and modal aspects (Fig. 4).

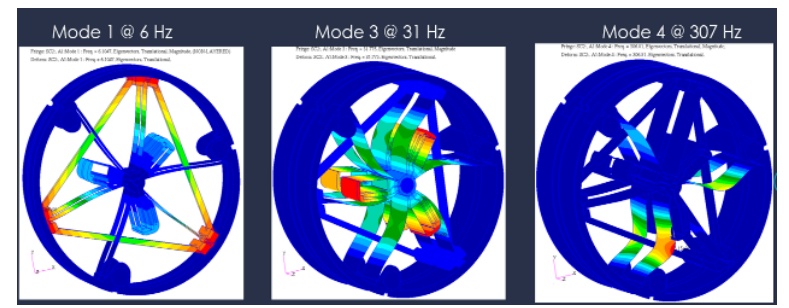

Fig. 4. Modal analysis with rotation blocked by LLD

Preloading was added to the simulation through a semi-analytical model. With Nastran, the real bow-tie tapered profile of the leaf springs was implemented on shell elements, which were connected by realistic rigid parts. The preload is performed by symmetrically adjusting the gaps of each stage during the assembly procedure then gluing.

\section{Main Pivot requirements}

The high level requirements for the pivot mounted in pairs with a mobile payload is:

- Angular range $\quad \pm 70^{\circ}$ (target for lifetime)

- Central shift $\quad<10 \mu \mathrm{m}$ (no load, max rotation)

- Angular stiffness Minimise (@90 $\left.90^{\circ}\right)$

- Payload mass $\quad 1.2 \mathrm{~kg}$

- Number of cycles 2.4 million

- Temperature range $-125^{\circ}$ to $+50^{\circ} \mathrm{C}$ (operational)

- Random vibration 18.4/12.2grms (X/Y\&Z) 
Table 1. Performances of the LAFP

\begin{tabular}{|l|c|l||}
\hline Property & Value & Unit \\
\hline \hline Torque @ 90 & 0.53 & $\mathrm{Nm}$ \\
\hline Natural stiffness @ 0 & 70 & $\mathrm{mNm} / \mathrm{rad}$ \\
\hline Natural stiffness @ 90 & 340 & $\mathrm{mNm} / \mathrm{rad}$ \\
\hline Transversal stiffness @ 0 & 832 & $\mathrm{kN} / \mathrm{m}$ \\
\hline Transversal stiffness @ 90 & 22 & $\mathrm{kN} / \mathrm{m}$ \\
\hline Axial stiffness @ 0 & 1076 & $\mathrm{kN} / \mathrm{m}$ \\
\hline Axial stiffness @ 90 & 125 & $\mathrm{kN} / \mathrm{m}$ \\
\hline Diameter & 120 & $\mathrm{~mm}$ \\
\hline
\end{tabular}

In the end, results from both FEM software are in good agreement, see Fig. 5. Performance of the pivot satisfied the specifications, regardless of the decrease in lateral stiffness that is typical for large deformation flexure pivots.
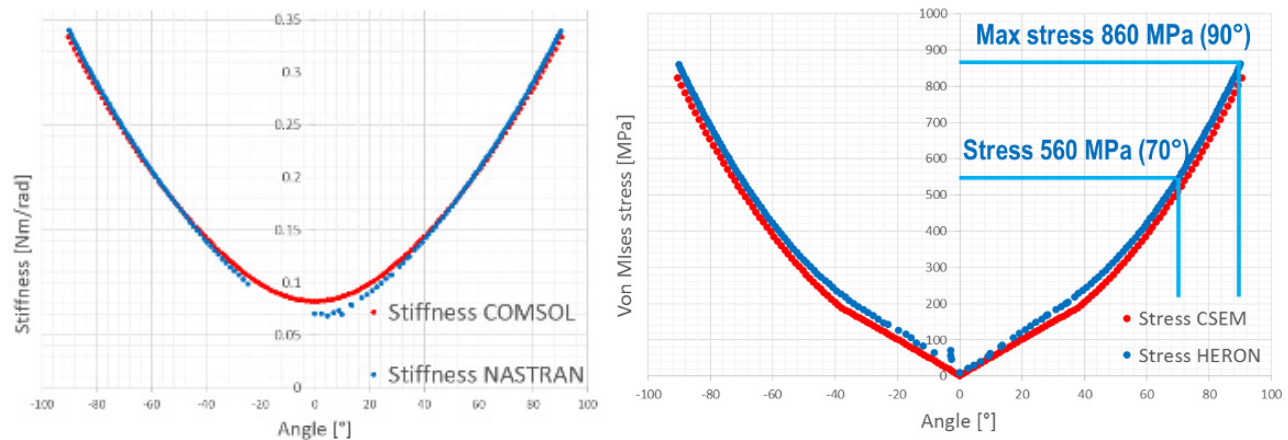

Fig. 5. LAFP stiffness and stress FEM comparison

\section{Detailed FEM analysis with test bench}

Additional simulations were performed concerning dynamic (shocks, random and sine vibrations) and thermal aspects. Also, dynamic simulations at the system level were made in order to size a launch locking device.

\subsection{Detailed FEM analysis of pivot alone}

The FEM consists of 425,898 nodes and 390,246 elements (solids \& shells). Material is steel with properties $\mathrm{E}=195 \mathrm{Gpa}, \mathrm{v}=0.33$, Density $=7800 \mathrm{Kg} / \mathrm{m} 3$ and $\mathrm{CTE}=10 \times 10^{\wedge}-6$ $\mathrm{m} / \mathrm{m} * \mathrm{~K}$.
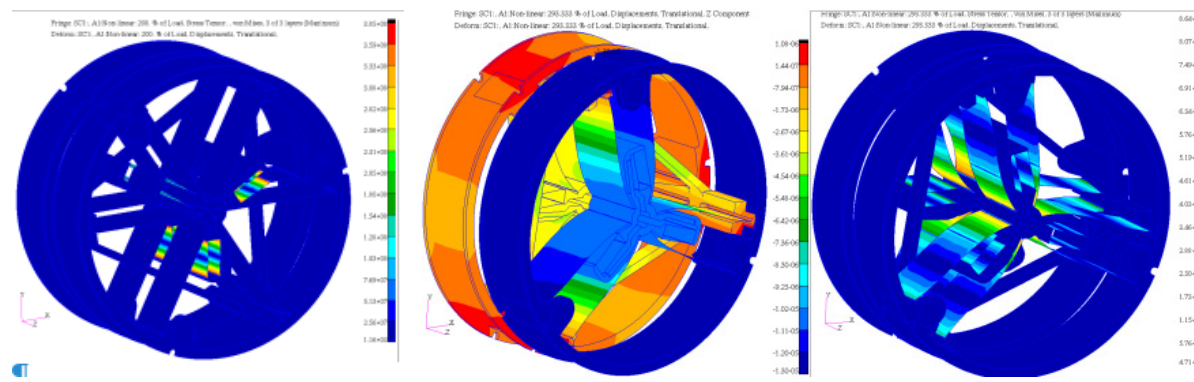

Fig. 6. Pivot preload stress $0^{\circ}$ (left), Shift (middle) and Von Mises stress at +90 degrees 
In order to simulate the preloading (Fig. 3b) due to the bonding action, Nastran "contact pairs" were used. A contact pair may be "touching" (it can separate) or "glued" (it cannot separate once the two surfaces come into contact). The contact pair also prevents penetration of one surface into the other. For the bonding action, two sequential steps were defined:

- Step 1: Close Gap (contact is "touching")

- Step 2: Release closing force (contact is "glued")
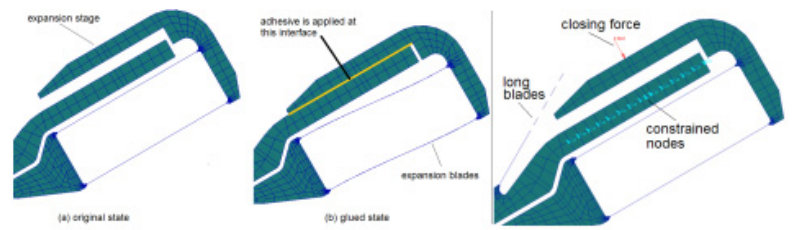

Fig. 7. Bonding and Boundary Conditions

These steps are always the starting point of any analysis, whether this is the operational case (rotation to $\pm 90^{\circ}$ ) or the dynamic load cases (modal analysis, sinusoidal vibration, random vibration, etc). Figure 6 (left), shows the preload (or residual) Von Mises stresses at the end of Step 2 (Release). Step 3 was defined as the Operational Case: prescribed torque for rotation to $\pm 90^{\circ}$.

\subsection{Thermal analysis of pivot with EM Test bench}

To understand the behaviour the pivot in the test bench during the thermal cycling tests, a thermal analysis was performed to estimate the time it will take for the pivot to reach its target temperature of $-140^{\circ} \mathrm{C}$ from Room Temperature $\left(+20^{\circ} \mathrm{C}\right)$. The model of Figure 6 was used for the pivot. The support table and the 3 washers (joining the pivot to the table) were modelled with solid elements shown in Figure 8.

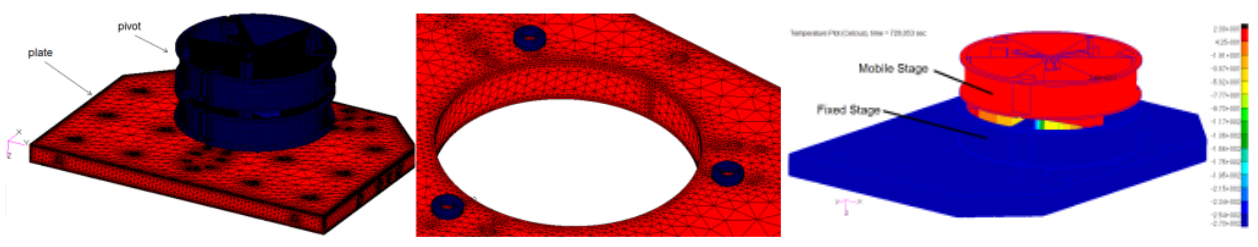

Fig. 8. Thermal Model details and thermal analysis results at $t=200$ hours

A cooling flux of $150 \mathrm{Watt}$ is applied on one side of the plate. This cooling flux is considered to be the only mechanism of cooling in the test setup. Observations are that the fixed stage, close to the plate, reaches absolute zero $\left(-273.15^{\circ} \mathrm{C}\right)$ within 4.56 hours and the mobile stage remains at $+20^{\circ} \mathrm{C}$. The setup (plate+pivot) will need to be cooled down first, by some other mechanism, e.g. convection with Nitrogen followed by the $150 \mathrm{Watt}$ cooling.

\subsection{Mechanical analysis of vibration test bench}

In order to better predict the behaviour of the pivot during test and consequently in real environment, analyses were performed of the pivot installed in the vibration test bench. The test bench was modelled with solid elements and the model shown in Figure 6 was used for the pivots. 


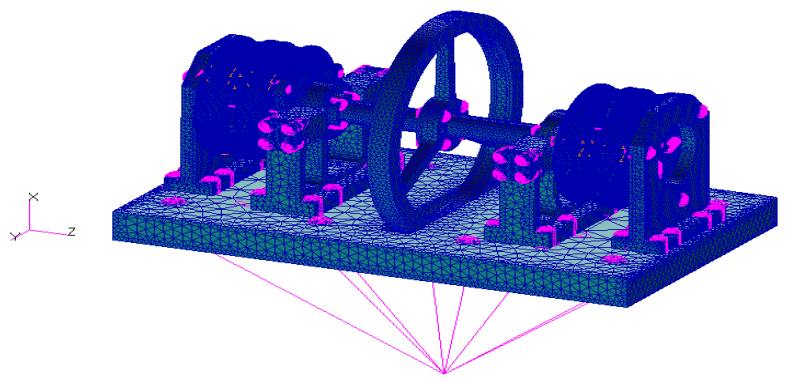

Fig. 9. FEM of the Test bench with pivots

The vibration table is simulated as an independent node, below the bench plate. This node is attached to the 8 fastener holes of the bench table via an RBE2 rigid constraint transferring all six (6) degrees of freedom. In all dynamic analyses (sine, random, shock), this node is used for applying prescribed displacement or acceleration on to the test bench. The analyses show a different behavior of the pivot in the test bench under random vibration loads compared to the analyses of the pivot by itself. Stresses are higher, especially during $\mathrm{X}$-axis vibration.

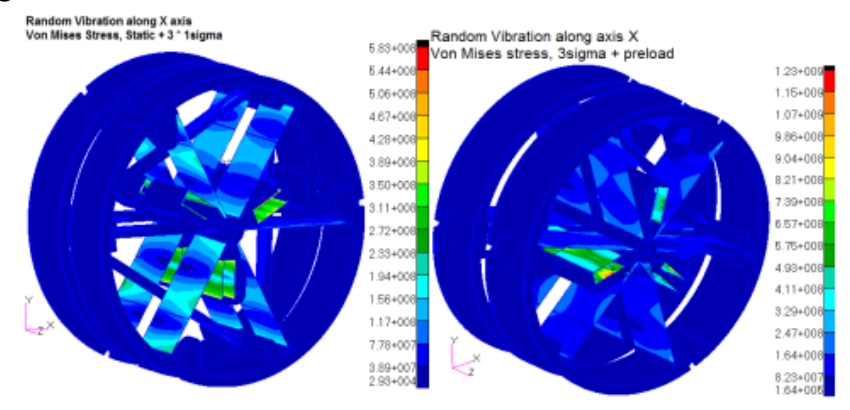

Fig. 10. Combined (preload $+3 \sigma$ ) $\mathrm{X}$ axis random vibration -pivot alone \& pivot in the bench

\section{EXPERIMENTAL SETUP}

\subsection{EM Test Bench}

The pivot rotary stiffness was measured at component level using a Kistler torque sensor and compared with the FEM values which show a very good correlation. The average maximum torque of both pivots at $\pm 90^{\circ}$ is $0.52 \mathrm{Nm}$ (FEM: $0.53 \mathrm{Nm}$ ). The two pivots were then integrated in the Engineering Model (EM) test bench for functional and performance tests.

The EM test bench (Fig. 11) illustrates a typical mechanism configuration with its representative dummy payload for mass and inertia in the form of a wheel on a shaft between two pivots. The objective of the test bench is to verify pivot performance notably the parasitic lateral (or centre) shift, angular range and angular stiffness. The same test bench is used for the TVAC (Thermal Vacuum and Cycling) and Lifetime tests.

\subsection{EM Functional Test Results}

Lateral shifts on EM test bench are measured through internally mounted eddy current sensors on a sensor ring which is fixed to the test bench. Their target is a cylinder directly connected to the shaft on which the pivots and the inertial wheel are connected. External 
Micro-Epsilon laser sensors provide a second reference for the lateral shift errors. Results in Table 2 indicate that the lateral shift error for $\pm 70^{\circ}$ pivot rotation is $\pm 35 \mu \mathrm{m}$ which is above the requirements of $<10 \mu \mathrm{m}$ but remains stable before and after the vibration tests.

Table 2. Lateral shift comparison before/after vibration

\begin{tabular}{|c|c|c|c|c|c|c|}
\hline & \multicolumn{4}{|c|}{ SNO2 (Top +Z) } & \multirow{2}{*}{\multicolumn{2}{|c|}{$\begin{array}{l}\text { SN01 (Botfom -Z) } \\
\mu \text { Epsilon (laser) }\end{array}$}} \\
\hline & Eddy curre & t sensor & $\mu$ Epsilo & (Iaser) & & \\
\hline & $x$ & $\mathbf{Y}$ & $x$ & $Y$ & $x$ & $Y$ \\
\hline Before Vib. & $\pm 6 \mu \mathrm{m}$ & $=43 \mu \mathrm{m}$ & $\pm 8 \mu \mathrm{m}$ & $\pm 41 \mu \mathrm{m}$ & $\pm 41 \mu \mathrm{m}$ & $\pm 40 \mu \mathrm{m}$ \\
\hline After Vib. & $\pm 37 \mu \mathrm{m}$ & $=32 \mu \mathrm{m}$ & $\pm 32 \mu \mathrm{m}$ & $\pm 40 \mu \mathrm{m}$ & $\pm 32 \mu \mathrm{m}$ & $\pm 33 \mu \mathrm{m}$ \\
\hline
\end{tabular}

\subsection{Vibration Test Bench and Test results}

Following the functional tests, the pivots were transferred and mounted on the vibration test bench which has a similar configuration to the EM performance test bench. It is a dedicated test bench incorporating a representative payload with a dummy launch locking device (LLD) but without the actuator. The high level sine and random vibration tests were performed in all directions with the sequence being Y-direction, Z-direction and finally Xdirection. The tested levels for sinusoidal vibration was $24 \mathrm{~g}(20-100 \mathrm{~Hz})$ and $12.2 \mathrm{gRMS}$ for the random vibrations. Test details found in Ref [1]. Following the vibration tests, the pivots were disassembled from the test bench and remounted in the EM Test bench for TVAC and Lifetime tests.

\subsection{TVAC and Lifetime Tests at Cedrat Technologies}

The TVAC tests are performed in a chamber (Fig. 11) comprised of:

- Cryo compatible Eddy current sensors (lateral shift)

- Angular position knowledge using reed switches mounted on wheel

- Renishaw rotary encoder read head is removed (not compatible with cryo temp.)

-Functional operation of voice-coil actuator controlled in open loop
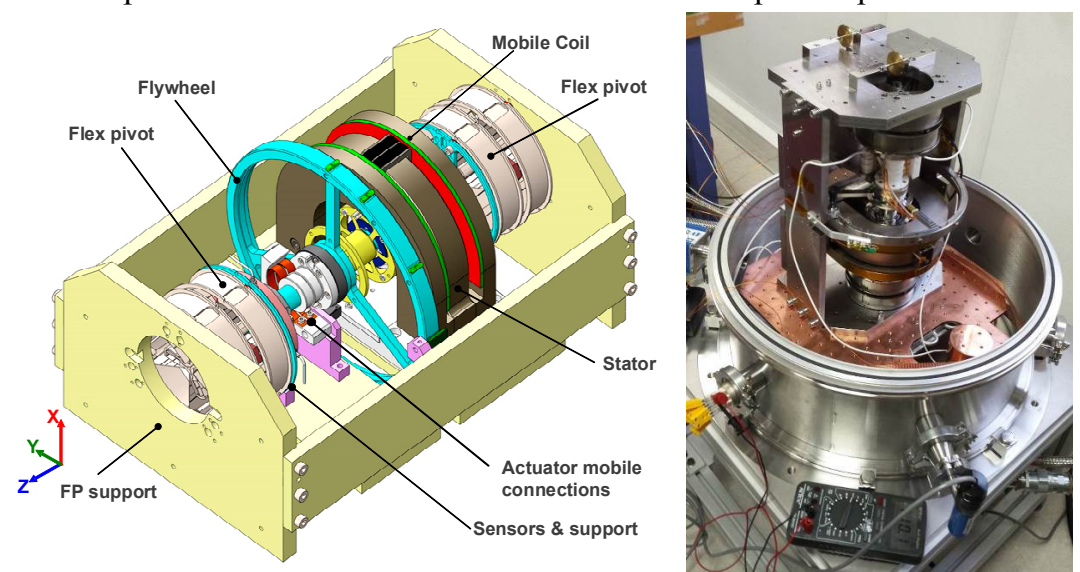

Fig. 11. EM mounted in TVAC chamber in vertical configuration

An accelerated lifetime test approach at ambient temperature will be performed where the flexural pivot mechanism assembly shall be actuated at $1 \mathrm{~Hz}$. The test duration will be between 30 to 40 days to achieve the target of 3.1 million cycles. 


\section{CONCLUSION}

The performance tests have confirmed a lateral shift of less than $\pm 35 \mu \mathrm{m}$ over an angular range of $\pm 90^{\circ}$. The pivots have been successfully tested and survived vibration loads for high level sine at $24 \mathrm{~g}$ and random vibration at $12 \mathrm{grms}$ in all three directions. Even though one of the pivot blades was damaged due to misalignment from an assembly error, the test campaign continued as planned.

Thanks to the LAFP concept, it is now possible to overcome one of the major historic drawbacks of the rotary compliant mechanisms, short angular strokes. Indeed, it reaches beyond state-of-the-art performances in terms of stroke and guiding quality. Once all the validations are passed, this mechanism will be qualified for concrete use in the frame of a future space science missions. The patented design is suitable for various applications in different domains.

\section{REFERENCES}

1. P. Spanoudakis, L. Kiener, F, Cosandier, P. Schwab, L. Giriens, J. Kruis, D. Grivon, G. Psoni, C. Vrettos, N. Bencheikh. Large angle flexure pivot development for future science payloads, $18^{\text {th }}$ ESMATS, (2019)

2. F. Cosandier, P. Spanoudakis, P. Schwab, L. Kiener, J. Kruis, L. Giriens, C. Vrettos, G. Psoni. Large angle flexure pivot development for high accuracy positioning of optical payloads. euspen's 19th International Conference \& Exhibition, (2019)

3. S. Henein, P. Spanoudakis, S. Droz, L. Myklebust, E. Onillon, Flexure pivot for aerospace mechanisms, $10^{\text {th }}$ ESMATS, (2003)

4. R. Fowler, A. Maselli, P. Pluimers, S.P. Magleby, L. Howell, Flex-16: A largedisplacement monolithic compliant rotational hinge, Journal of Mechanism and Machine Theory 82 203-217, (2014)

5. K. Gunnink, R. Aarts, D. Brouwer, Performance optimization of large stroke flexure hinges for high stiffness and eigenfrequency, 28th ASPE - American Society for Precision Engineering, (2013)

Key words: compliant mechanism, large angle flexure pivot, space environment, precision opto-mechatronics, cryogenic 